\title{
Microglia and astroglia proliferation within the normal appearing white matter in histologically active and inactive multiple sclerosis
}

\author{
Przemysław Nowacki, Dorota Koziarska, Marta Masztalewicz \\ Department of Neurology, Pomeranian Medical University, Szczecin, Poland
}

\begin{abstract}
Introduction: Multiple sclerosis (MS) is an autoimmune, neurodegenerative disease with the involvement of white and grey matter. Histopathological abnormalities can be also identified in normal appearing white matter (NAWM). Aim of the study: To compare the micro- and astroglia proliferation within NAWM in MS cases with and without inflammatory activity within central nervous system (CNS).

Material and methods: In post-mortem brain examinations we investigated 13 MS cases and 5 individuals who suddenly died for cardiological reasons without neurological comorbidities. We analysed micro- and astroglia proliferation within NAWM in histologically active and inactive MS cases in relation to the control group. To avoid the possible influence of the modern disease modifying treatment (DMT) on NAWM histopathological appearance, we decided to investigate the individuals who died before the modern DMT was introduced in Poland. The morphometric analysis of micro- and astrogliosis was carried out at magnification $10 \times 10$ (microscopic lens $\times$ oculars $=$ magnification 100x). The fields were expressed in $\mathrm{mm}^{2}$; each field $=0.364 \mathrm{~mm}^{2}$. In total, 775 fields of NAWM and 420 fields of white matter in the control group were morphometrically assessed.

Results: Significantly higher microglia proliferation appeared both in MS active and inactive cases compared to controls, while there were no differences between active and inactive cases. There was a significantly higher proliferation within NAWM of active MS cases compared to the inactive ones and controls as well as between inactive cases and controls. There were no correlations between microglia and astrocytes proliferation either in active or inactive MS groups.

Conclusions: Our results suggest that within MS NAWM, which appears to be involved at the very least, intense diffuse micro- and astroglia reactions occur. Possibly, microglia rather support proinflammatory mechanisms, whereas astrocytes seem to be more neuroprotective. Diffuse microglia proliferation indicates that CNS immune system is chronically activated within the whole CNS.
\end{abstract}

Key words: multiple sclerosis, normal appearing white matter, astroglia proliferation, microglia proliferation.

\section{Introduction}

Multiple sclerosis (MS) is now no longer considered to be a disease limited to classic central nervous system (CNS) white matter plaques, which can be readily identified either radiologically or at autopsy [20]. Neuroimaging of live patients has had a major impact not only on early diagnosis and management, but also on the evolving recog-

\section{Communicating author}

Prof. Przemysław Nowacki, Department of Neurology, Pomeranian Medical University, Unii Lubelskiej 1, 71-252 Szczecin, Poland, e-mail: przemyslaw.nowacki@hotmail.com 
nition of the effects of the disease on the entire CNS $[29,36]$. In daily radiological practice, a simplified analysis has been introduced, limited to two main changes: active and inactive MS lesions (plaques) and extra-plaque zones. Within the CNS of MS patients three main zones are distinguishable from the histopathological point of view: plaques (acute active, chronic active, inactive, and shadow plaques), diffusely abnormal white matter and the so-called normal appearing white matter (NAWM) $[7,9,15,25]$. Indeed, macroscopically and histologically at lower magnification, the majority of NAWM appears normal, but histopathological abnormalities can be identified in NAWM at higher magnification [20]. NAWM abnormalities can also be detected using non-conventional magnetic resonance imaging $[13,14,25]$. Recent studies on NAWM have revealed correlations between histopathological changes and magnetic resonance abnormalities [19]. These studies have brought about interest in the pathological meaning and the clinical importance of NAWM in MS $[3,18]$. The neuropathological changes in MS NAWM may consist of blood-brain-barrier (BBB) dysfunction, perivascular inflammatory infiltrations, myelin damage, and axonal disruption or even loss [6]. Micro- and astrogliosis are also observed within NAWM. Glial cells produce high levels of nitric oxide in response to proinflammatory stimuli, leading to nervous tissue damage in MS [1]. Activated microglia may also express matrix metalloproteinases and tumor necrosis factor $\alpha$ (TNF- $\alpha$ ) that can facilitate CNS involvement by inflammation $[23,24,30,40]$. Many findings concern the microglia and astrocytes reactivity in the active stage of the disease, especially within MS plaques. Abnormalities documented in MS NAWM had also been initially attributed to active focal lesions. Nonetheless, the behaviour of NAWM micro- and astrocytosis in active and inactive MS cases remains less known.

The purpose of our study was to compare the micro- and astroglia proliferation within NAWM in MS cases with and without inflammatory activity within CNS. To avoid the possible influence of the modern disease modifying treatment (DMT) on NAWM histopathological appearance, we decided to investigate the individuals who died before the modern DMT was introduced in Poland.

\section{Material and methods Material}

The post-mortem brain examination was done on 13 cases, aged 29 to 59 years (median 42 years; 8 women, 5 men), deceased due to MS between 1966 and 1977. Duration of the disease ranged between 6 and 24 years. Based on retrospective analysis of clinical data we estimated that the neurological deficit reached around 9 EDSS. At that time, MS relapses, also in our patients, were treated with adrenocorticotropic hormone (ACTH), the most established and validated option for MS relapse [26]. The patients had died before a present-day neuroimaging and modern DMT were implemented in Poland.

Post-mortem final diagnosis was done by an experienced neuropathologist. In each case included in the study the histopathological changes were located both infra- and supratentorially, the latter predominantly within the white matter, around the lateral ventricles. The main neuropathological changes were as follows: active and inactive MS plaques, diffused abnormal white matter (DAWM) and so-called NAWM. The acute active plaques contained robust immune cell infiltrates, demyelination, parenchymal oedema and axons damage. Within the chronic active (burning) plaques, inflammation continued along the border, while the core has become hypocellular. Demyelination, features of remyelination as well as axonal destruction were also observed. In the chronic inactive plaques inflammation was absent; they were almost completely acellular, axonal density was reduced while gliosis appeared to be intensive. MS plaques were also found within the cortex and deep grey matter. DAWM usually adhered to the plaques. It was characterized by poorly defined boundaries. Diffuse pallor or loss of myelin, axons destruction or loss, astro/microglia proliferation were the hallmark of DAWM. Perivascular T-cell/B-cell infiltrations, sometimes as perivascular cuffings were observed in MS active cases. MS plaques surrounded by DAWM were predominantly located near the lateral ventricles. White matter regions macroscopically and in microscopical lower power appearing normal were considered as NAWM, however palled myelin, interrupted axons, astro- and microglia proliferations as well as small perivascular inflammation in active cases were observed at higher magnification.

In all examined cases, spinal cord MS changes were observed, including MS active and inactive 
plaques as well as myelin and axonal damage. MS plaques were located both in white and grey matter, at all spinal levels, especially in the cervical part. Myelin pallor or loss was also observed in spinal roots. In some cases small inflammatory infiltrates appeared in leptomeninges.

Based on clinical manifestations and neuropathological examination, main other CNS inflammatory demyelinating diseases, in particular neuromyelitis optica spectrum and acute demyelinating encephalomyelitis were excluded. In all patients, the relapsing remitting with consecutive secondary progressive MS course has been documented. They had minimal, if any, visual disturbances. Cerebrospinal fluid assessment had not revealed pleocytosis. In all cases pathological foci (plaques, DAWM) evidently extended supratentorially. The plaques, including chronic inactive ones had not demonstrated necrotic features, inflammatory cells spectrum was not consistent with MS inflammation. In our opinion, above listed features justified us to establish MS diagnosis in our patients.

At that time, MS relapses, also in our patients, were treated with adrenocorticotropic hormone (ACTH), the most established and validated option for MS relapse [26]. The control group consisted of brains taken from 5 individuals aged 47-65 years who suddenly died for cardiological reasons without neurological comorbidities. The Pomeranian Medical University Ethics Committee approved the study protocol, which conformed to the ethical guidelines of the Declaration of Helsinki.

\section{Methods}

\section{Neuropathological examination}

The detailed neuropathological examination was done based on analysis of coronal large-hemispheric or double-hemispheric slices including caudate nuclei, putamen, pallidum, thalamus, and additionally based on sections from pons, medulla oblongata and cerebellum, including dentate nuclei. Paraffin-embedded specimens were initially stained with hematoxylin and eosin, Heidenhain's, Holzer's, Bielschowsky and van Gieson methods. Recently selected tissue fragments were immunostained with GFAP (Rabbit (pAB) Astrocytic GFAP 1 : 2000 St (E)* Z0334; Dako), CD68 antibodies (CD68 Mouse (mAB) microglia/macrophages 1 : 100 St (E) M0814; Dako), TCD3 (Polyclonal Rabbit Anti-Human CD3; code: IR503;
Dako; TCD4 (Monoclonal Mouse Anti-Human CD4; Clone: 4B12, Code: IR649; Dako) and TCD8 (Monoclonal Mouse Anti-Human CD8; Clone: C8/144B, Code: IR623; Dako).

\section{Microglia and astroglia proliferation within NAWM}

The morphometric analysis of micro- and astroglia proliferation within NAWM (Fig. 1C,D) was done with the cellSens imaging software from Olympus, version 1.17 .

White matter regions macroscopically and in microscopical lower power appearing normal were considered as NAWM (Fig. 1A). The densitometric profile of NAWM myelin, assessed with RGB scale was as follows: for $\mathrm{R} M e=32(\mathrm{Q} 1=15-\mathrm{Q} 3=58)$, for $\mathrm{G} M e=50(\mathrm{Q} 1=31-\mathrm{Q} 3=79)$, for $\mathrm{B} M e=83$ $(\mathrm{Q} 1=60-\mathrm{Q} 3=112$ pts $)$ (Fig. 1B). NAWM was defined as a region located at least $10 \mathrm{~mm}$ apart from MS lesions. Three tissue areas of NAWM and three areas of controls white matter were measured in each case. The number of astrocytes and microglia cells within the consecutive 10 to 20 fields for each of three NAWM tissue areas, and 20 fields for each of three white matter areas taken from control individuals was calculated. The morphometric analysis was carried out at magnification $10 \times 10$ (microscopic lens $\times$ oculars $=$ magnification $100 \times$ ). The fields were expressed in $\mathrm{mm}^{2}$; each field $=0.364 \mathrm{~mm}^{2}$. In total, 775 fields of NAWM and 420 fields of white matter in the control group were morphometrically assessed.

\section{Histological MS activity classification}

The material was divided into two groups. Group I (histologically active MS group) consisted of 8 cases and demonstrated acute active and chronically active MS lesions. Active lesions were infiltrated by macrophages filled with myelin products and T-cells located within the whole lesion. In chronically active lesions macrophages and T-cells were located at the peripheral zones of the lesion. Perivascular hematogenic infiltrates were also present in acute and chronic active lesions [18]. Group II (histologically inactive MS group) included 5 cases without acute and chronically active MS lesions and without hematogenic infiltrates either within MS plaques or within the other investigated CNS regions. 

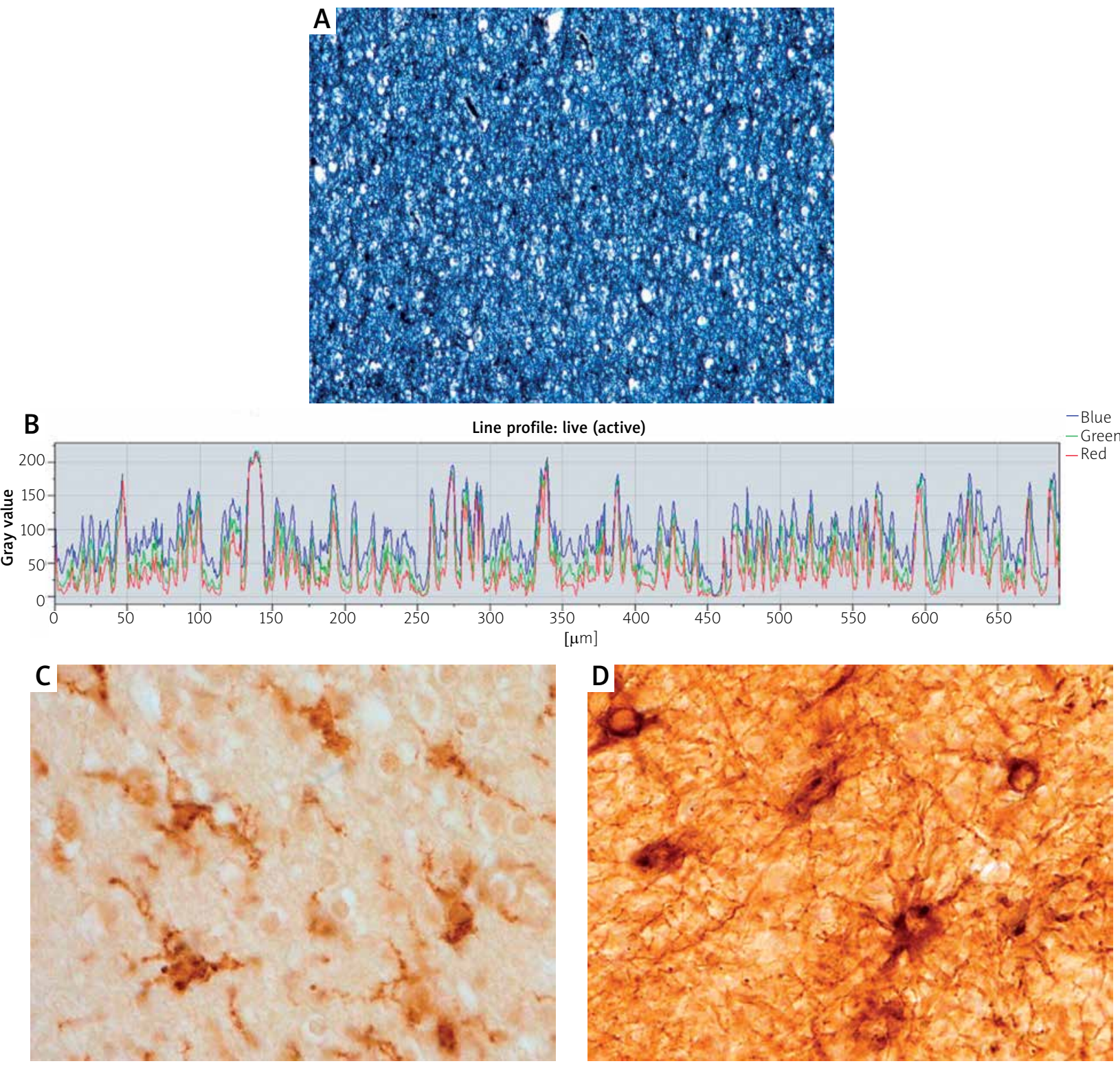

Fig. 1. A) NAWM - normal appearing myelin in lower power, Heidenhain, 100×. B) NAWM RGB profile. C) Microglia cells, anti-CD68 antibody, 400x. D) Astrocytes, anti-GFAP antibody, 400×.

\section{Statistical analysis}

The statistical null hypothesis was that microand astroglia proliferation within NAWM in histologically active MS cases does not significantly differ from micro- and astroglia proliferation in inactive MS cases. The alternative hypothesis was that micro- and astroglia proliferation within NAWM in histologically active MS cases significantly differs from micro- and astroglia proliferation in inactive MS cases. To assess the equality of variances for variables, the Levene's test was used before a comparison of means. The test has shown significance $(p<0.05)$. For this reason the variables were presented as median (Me), the first quartile (Q1) and the third quartile (Q3). Because of the non-normality of the distributions between variables, the data were compared between groups I, II and control using the nonparametric Kruskal-Wallis one-way analysis of variance for continuous variables. Correlations between quantitative variables were studied with $r$-Pearson correlation. $P$ values smaller or 
equal to 0.05 were considered to indicate statistical significance. All statistical analyses were performed with STATISTICA 12.5 pl.

\section{Results}

\section{Microglia proliferation}

In histologically active MS cases (group I), the number of microglia cells within NAWM ranged from 129 to 214 per analysed field (Me = 172 cells; Q1 = 155, Q3 = 183 cells). In histologically inactive MS cases (group II), the number of microglia cells within NAWM ranged from 129 to 210 cells per field (Me = 184 cells; $\mathrm{Q} 1=173, \mathrm{Q} 3=193.5)$. In the control cases, the microglia cells in consecutive fields ranged from 22 to $47(M e=33 ; \mathrm{Q} 1=29, \mathrm{Q} 3=38)$. The difference between microglia proliferation within NAWM of active and inactive cases was presented in Fig. 2. Significantly higher microglia proliferation appeared both in MS active and inactive cases compared to controls, while there were no differences between active and inactive cases.

\section{Astroglia proliferation}

In consecutive NAWM fields of active MS cases, the astrocytes count ranged from 50 to 73 cells (Me = 65.5; Q1 = 57, Q3 = 69.5 cells $)$. In inactive MS cases, the number of astrocytes ranged from 33 to
71 per analysed field $(\mathrm{Me}=49 ; \mathrm{Q} 1=44, \mathrm{Q} 3=53)$. Within the consecutive fields of control individuals, the number of astrocytes was 16 to 49 ( $M e=32$; $\mathrm{Q} 1=27, \mathrm{Q} 3=37)$. The difference between astroglia proliferation within NAWM of active and inactive cases was depicted in Fig. 3. There was a significantly higher proliferation within NAWM of active MS cases compared to the inactive ones and controls as well as between inactive cases and controls.

There were no correlations between microglia and astrocytes counts either in active or inactive MS groups ( $r$-Pearson correlation; $p=0.31$ and 0.76 respectively).

The number of microglia cells and astrocytes differed significantly between some fields of the same case and between cases (Fig. 4A-D) (ANOVA Kruskal-Wallis test, $p<0.05)$. There were no substantial differences concerning the number of micro- and astrocytes in analysed fields of the control group. Within NAWM, the tendency to agglomeration of astrocytes near to the vessels was observed (Fig. 5A). The perivascular space was often separated from the nervous tissue by dense limbus consisted of fibrillary astrogliosis and astrocytic feet (Fig. 5B).

Both in active and inactive cases myelin sheaths within NAWM were irregular, vacuolated and dissected (Fig. 5C). Irregularly swollen axons were also
A

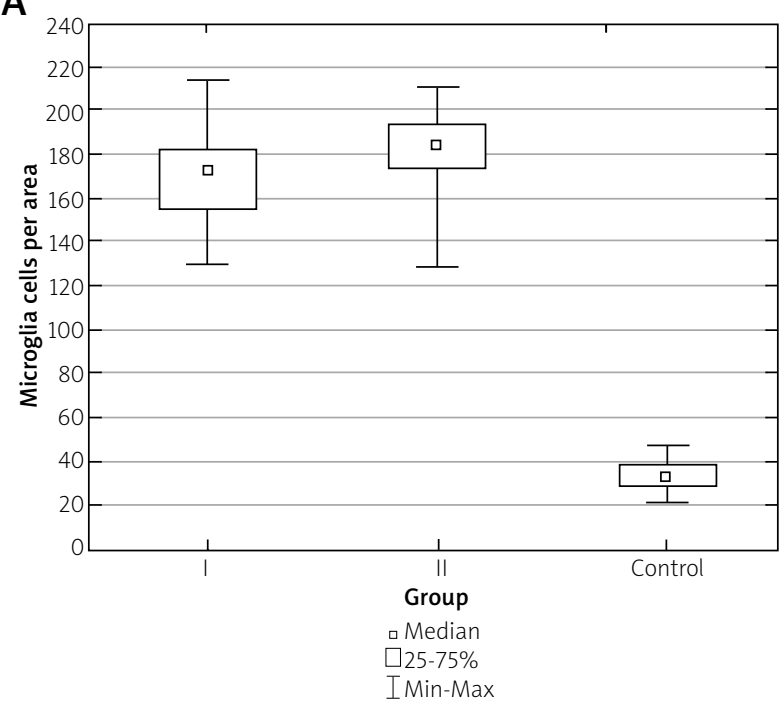

Fig. 2. The difference between microglia proliferation within NAWM of active and inactive cases and within the white matter of control cases.

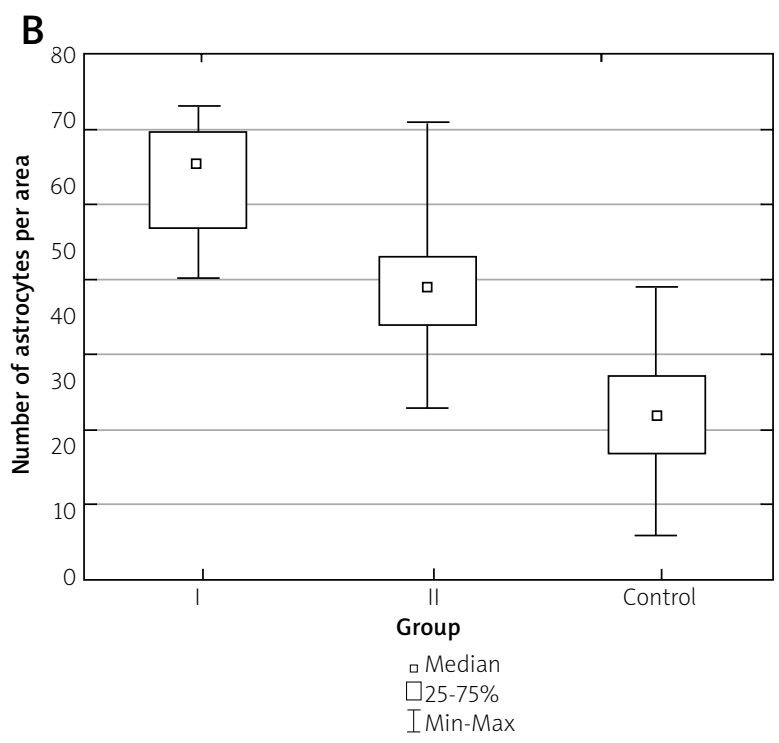

Fig. 3. The difference between astroglia proliferation within NAWM of active and inactive cases and within the control cases. 

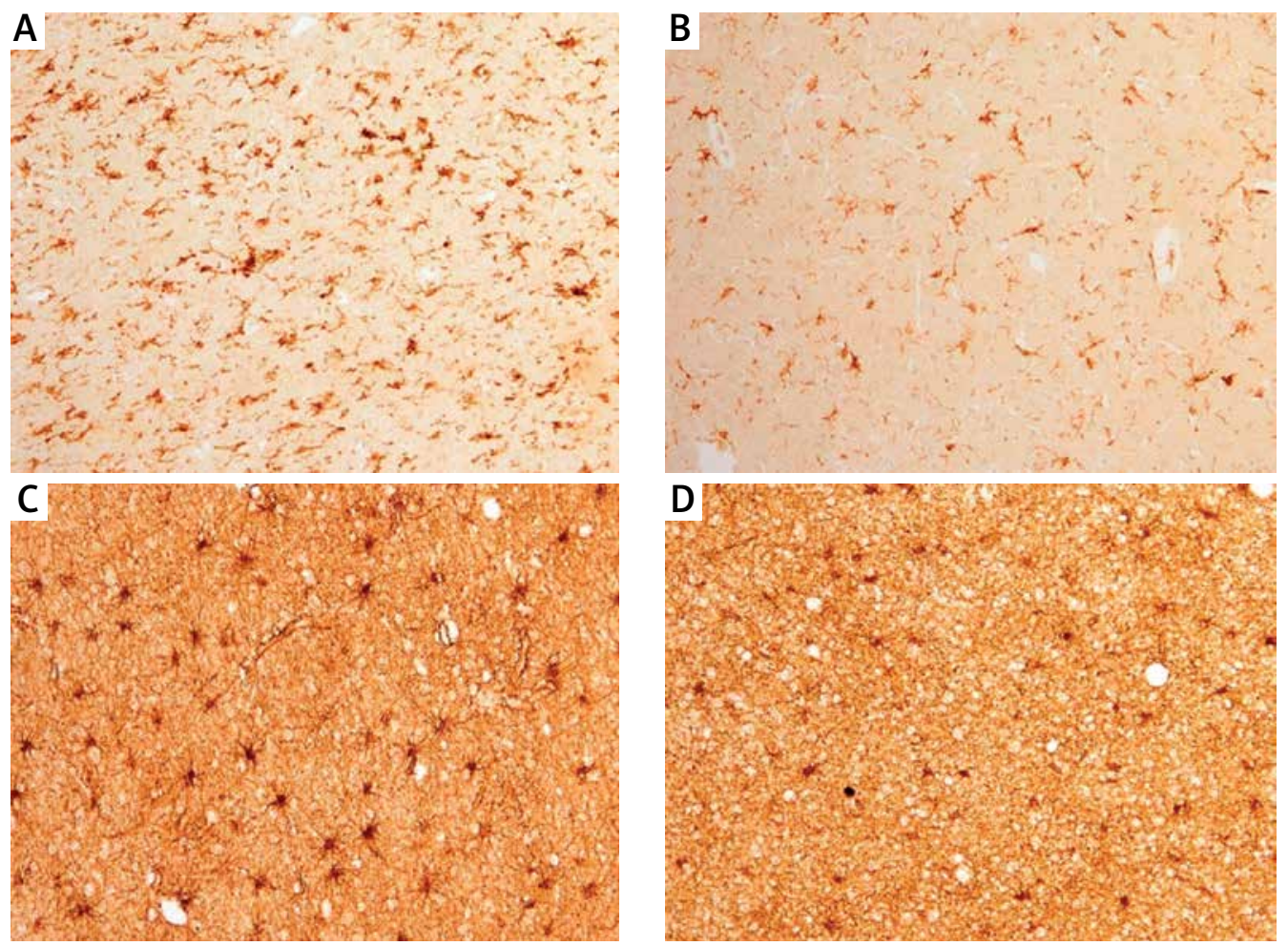

Fig. 4. Evident micro- (A) and astrogliosis (C) vs. relatively smaller micro- (B) and astrogliosis (D) in the same case.

observed (Fig. 5D, E). Sometimes axons were interrupted.

\section{Discussion}

In MS, the CNS is typically infiltrated by activated T lymphocytes, especially by CD3+, CD4+ and CD8+ subpopulations and less intensively by B-cells $[4,21,37]$. The microglia and astrocytes contribute to the cellular component of MS pathology as well. Local activation of microglia and astroglia, infiltration of activated macrophages and $\mathrm{T}$ cells, active degradation of myelin and damage to axons are the hallmarks of active and chronically active MS plaques [33]. A role of the glial cells as the antigen presenting in MS lesions was documented [16]. It is suggested that astroglia activation may exacerbate MS inflammation and demyelination within MS lesions [4,27].

Besides MS plaques, also within NAWM gliosis, perivascular infiltrates or myelin damage, including demyelination may be present. Activation of microglia, upregulation of osteopontin alfa B-cristallin and transcription factors involved in $\mathrm{MHC}$ expression were observed $[8,10,32]$. The relationship between microglia activation and axonal damage has been described within NAWM regions located near the plaques [20]. Aforementioned abnormalities documented in MS NAWM are usually also attributed to active MS cases. Little is known about the role of microglia and astroglia in NAWM of MS cases without hematogenic activity (inactive MS cases).

We decided to analyse the intensity of microand astrogliosis within NAWM in such cases. We have observed a significantly higher microglia reaction within NAWM both in histologically active and inactive cases as compared to controls, whereas there were no significant differences in intensity of microglia reaction between active and inactive cases. Regarding pronounced microglia proliferation within NAWM even in MS cases without hematogenic inflammation, we assume that the proliferative activity of microglia is maintained after extinction of inflammatory activity within the plaques and diffusely abnormal white matter. Considering that our patients were not treated with modern DMT, such massive participation of microglia even in histologically inactive MS cases might reflect a permanent response to "silent" pathogenic factor/factors. Thus, possibly inactive MS cases actually remain the 

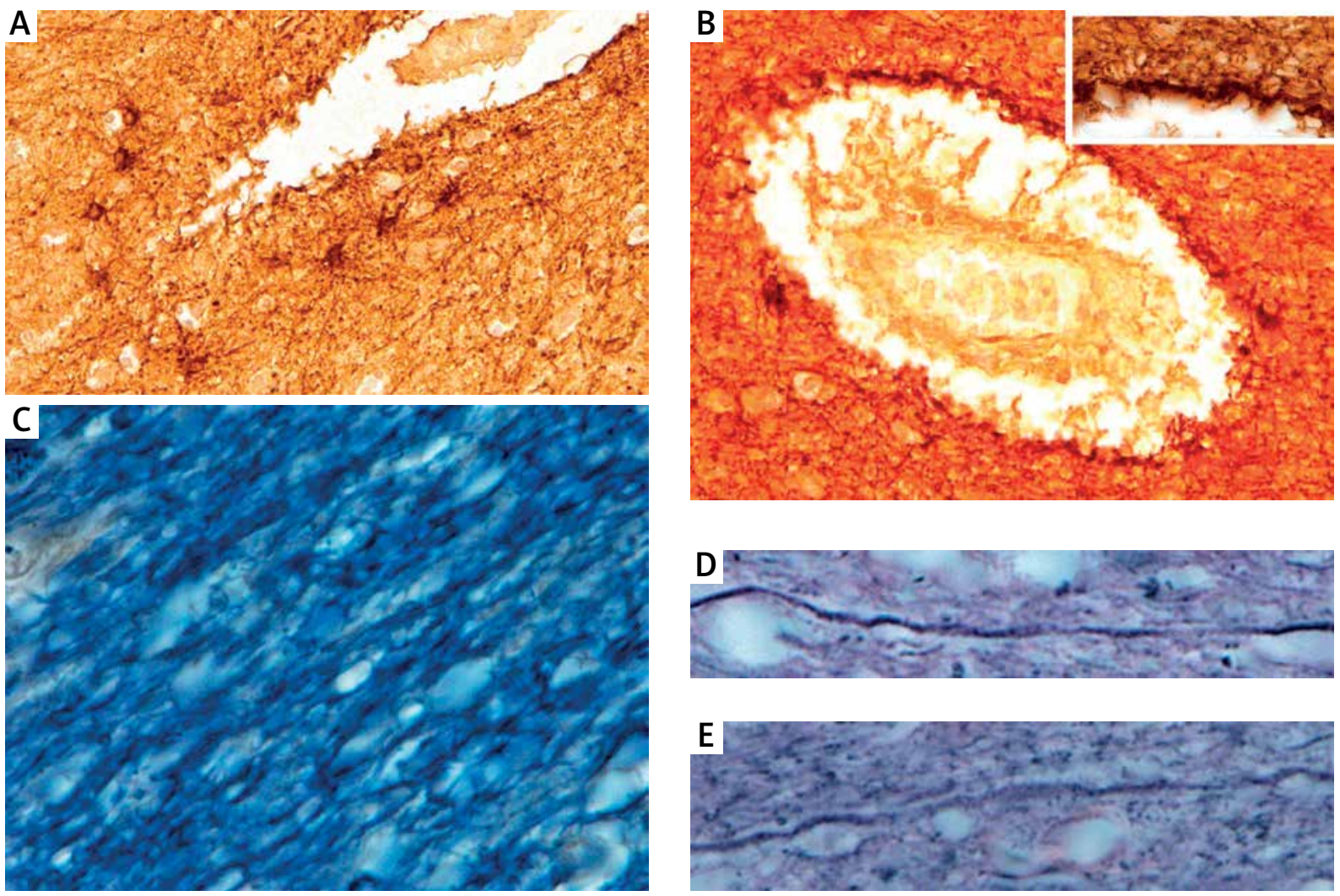

Fig. 5. A) Concentration of astrocytes in the vicinity of the vessel, anti-GFAP antibody, 100x. B) Astroglial fibrillary limbus separating perivascular space and white matter, anti-GFAP antibody, 100× (upper right corner; 400×). C) Myelin vacuolation, Heidenhain, 1000×. D, E) Swollen axons, Bielschowsky, 1000×.

active ones. It was documented on experimental allergic encephalitis that microglia may be activated before the immune cells invasion into CNS [11]. Consequently, myelin and axons in NAWM may be impaired by both "extrinsic" hematogenic immunoreaction and "intrinsic" neuroimmune system activity, also in clinically and radiologically inactive MS cases. Myelin isolated from NAWM was phagocytosed more efficiently than myelin isolated from control brains [9]. The authors postulate that NAWM myelin in MS might precede phagocyte activation and subsequent demyelination in MS. Moreover, axonal lesion, admittedly smaller than in MS plaques and diffusely abnormal white matter, also characterize NAWM. Axonal lesion may be secondary to axons dissection within the plaques but also due to massive microglia activation in NAWM. Thus, microglial inflammation may be an early indicator of myelin and axonal injury within NAWM [11]. Concededly, it is proposed that microglia may prevent more profound and extensive NAWM damage [17] and seem to play a role in maintaining neuronal connectivity [38]. Our results suggest that a massive microglia proliferation within NAWM leads rather to damage than protection of this region. It was documented that microglia contribute to irreversible tissue damage during the chronic inflammatory response [12].

The role of astrogliosis within MS NAWM seems to be complex and remains unresolved. On the one hand, a role for the astrocytes as antigen presenting cells is postulated [16]. Furthermore, they are able to produce inducible nitric oxide synthase and, consequently, high expression of nitric oxide in response to a wide variety of proinflammatory and degenerative stimuli [31]. On the other side, astrocytes were found to be involved in preservation of recently denuded axons [16]. Through the ability to redistribute iron, which is crucial for myelin repair in MS, and stimulation of oligodendrocyte progenitor cells to attempt spontaneous remyelination, astrocytes might be involved in protective mechanisms within NAWM [5]. It is well known that the CNS is deprived of the 
lymphatic system, hence the drainage of interstitial fluid is constricted. Recently the unique phenomenon called "glymphatic system" has been revealed within the CNS, providing the transport from and to the cerebrospinal fluid [22,34]. It was indicated that perivascular astrocytes high with aquaporin-4 play the outstanding role in the function of the glia-dependent glymphatic system [28]. They facilitate glymphatic flow. This system is stricken in the elderly, especially in Alzheimer's disease, posttraumatic brain injury, stroke and metabolic disorders. The role of the glymphatic system in MS pathogenesis is not out of question and requires profound analysis.

\section{Limitations}

It would be interesting to know the proportion of reactive microglia and astrocyte compared to the total number of these cells (activated and not activated) in addition to the absolute number of reactive cells [39]. Unfortunately, because of technical aspects, we have not had possibilities to expose reactive forms of micro- and astroglia. Similarly, there were no opportunities to examine histological specimens by means of aquaporin-4 antibodies, but as it was abovementioned, careful analysis of clinical and histopathological data, allowed us to diagnose our patients as fulfilling MS criteria.

\section{Conclusions}

Our results based on post-mortem analysis of MS cases untreated with modern DMT suggest that within MS NAWM, which appears to be involved at the very least, intense diffuse micro- and astroglia reactions occur. Possibly, these two cell populations of different origin might have a diverse meaning in MS NAWM pathology. Microglia rather support proinflammatory mechanisms, whereas astrocytes seem to be more neuroprotective. Maybe on this account there were no correlations between astro- and microglia proliferation either in our active or inactive MS groups. Diffuse microglia proliferation supports the suggestions that the CNS immune system is chronically activated within the whole CNS [21].

The widespread abnormalities in MS NAWM appear to be clinically important because they correlate with disability progression and cognitive impairment in secondary progressive MS [2,35].

\section{Disclosure}

The authors report no conflict of interest.

\section{References}

1. Alper G, Heyman R, Wang L Multiple sclerosis and acute disseminated encephalomyelitis diagnosed in children after long-term follow-up: comparison of presenting features. Dev Med Child Neurol 2009; 51: 480-486.

2. Batista S, Alves C, d'Almeida OC, Afonso A, Félix-Morais R, Pereira J, Macário C, Sousa L, Castelo-Branco M, Santana I, Cunha L. Disconnection as a mechanism for social cognition impairment in multiple sclerosis. Neurology 2017; 89: 38-45.

3. Beaino W, Janssen B, Kooij G, van der Pol SM, van Het Hof B, van Horssen J, Windhorst AD, de Vries HE. Purinergic receptors P2Y12R and P2X7R: potential targets for PET imaging of microglia phenotypes in multiple sclerosis. J Neuroinflamm 2017; 14: 259.

4. Buschmann JP, Berger K, Awad H, Clarner T, Beyer C, Kipp M. Inflammatory response and chemokine expression in the white matter corpus callosum and gray matter cortex region during cuprizone-induced demyelination. J Mol Neurosci 2012; 48: 66-76.

5. Clemente D, Ortega MC, Melero-Jerez C. The effect of glia-glia interactions on oligodendrocyte precursor cell biology during development and in demyelinating diseases. Front Cell Neurosci 2013; 20: 268.

6. Eftekhari E, Hojjat SP, Vitorino R, Carroll TJ, Cantrell CG, Lee L, Taylor MW, Morrow SA, Benhabib H, Aviv RI, Kassner A. Normal appearing white matter permeability: a marker of inflammation and information processing speed deficit among relapsing remitting multiple sclerosis patients. Neuroradiology 2017; 59: 771-780.

7. Frischer JM, Weigand SD, Guo Y, Kale N, Parisi JE, Pirko I, Mandrekar J, Bramow S, Metz I, Brück W, Lassmann H, Lucchinetti CF. Clinical and pathological insights into the dynamic nature of the white matter multiple sclerosis plaque. Ann Neurol 2015; 78: 710-721.

8. Gobin SJ, Montagne L, Van Zutphen M, Van Der Valk P, Van Den Elsen PJ, De Groot CJ. Upregulation of transcription factors controlling MHC expression in multiple sclerosis lesions. Glia 2001; 36: 68-77.

9. Hendrickx DA, Schuurman KG, van Draanen M, Hamann J, Huitinga I. Enhanced uptake of multiple sclerosis-derived myelin by THP1 macrophages and primary human microglia. J Neuroinflamm 2014; 11: 64.

10. Hendrickx DA, van Eden CG, Schuurman KG. Hamann J, Huitinga I. Staining of HLA-DR, Iba1 and CD68 in human microglia reveals partially overlapping expression depending on cellular morphology and pathology. J Neuroimm 2017; 309: 12-22.

11. Howell OW, Rundle J, Garg A, Komada M, Brophy PJ, Reynolds R. Activated microglia mediate axoglial disruption that contributes to axonal injury in multiple sclerosis. J Neuropathol Exp Neurol 2010; 69: 1017-1033.

12. Jack C, Ruffini F, Bar-Or A, Antel JP. Microglia and multiple sclerosis. J Neurosci Res 2005; 81: 363-373.

13. Jonkman LE, Soriano AL, Amor S, Barkhof F, van der Valk P, Vrenken H, Geurts JJ. Can MS lesion stages be distinguished with MRI? A postmortem MRI and histopathology study. J Neurol 2015; 262: 1074-1080.

14. Kim SH, Kwak K, Joung A, Lee SH, Choi YH, Lee JM, Kim HJ. Diffusion tensor imaging of normal-appearing white matter in patients with neuromyelitis optica spectrum disorder and multiple sclerosis. Eur J Neurol 2017; 24: 966-973. 
15. Laule C, Pavlova V, Leung E, Zhao G, MacKay AL, Kozlowski P, Traboulsee AL, Li DK, Moore GR. Diffusely abnormal white matter in multiple sclerosis: further histologic studies provide evidence for a primary lipid abnormality with neurodegeneration. J Neuropathol Exp Neurol 2013; 72: 42-52.

16. Lee SC, Moore GR, Golenwsky G, Raine CS. Multiple sclerosis: a role for astroglia in active demyelination suggested by class II MHC expression and ultrastructural study. J Neuropathol Exp Neurol 1990; 49: 122-136.

17. Melief J, Schuurman KG, van de Garde MD, Smolders J, van Eijk M, Hamann J, Huitinga I. Microglia in normal appearing white matter of multiple sclerosis are alerted but immunosuppressed. Glia 2013; 61: 1848-1861.

18. Miller DH, Thompson AJ, Filippi M. Magnetic resonance studies of abnormalities in the normal appearing white matter and grey matter in multiple sclerosis. J Neurol 2003; 250: 1407-1419.

19. Moll NM, Rietsch AM, Thomas S, RansohoffAJ, Lee JC, Fox R, Chang A Ransohoff RM, Fisher E. Multiple sclerosis normal-appearing white matter: pathology-imaging correlations. Ann Neurol 2011; $70: 764$ 773.

20. Moore GR, Stadelmann-Nessler CH. Demyelinating diseases. In Love S, Perry A, Ironside J, Budka H (eds.). Greenfield's Neuropathology. Taylor \& Francis Group, India, Replica Press PVT. Ltd. 2015; pp. 1297-1412.

21. Mori F, Nisticò R, Mandolesi G, Piccinin S, Mango D, Kusayanagi H, Berretta N, Bergami A, Gentile A, Musella A, Nicoletti CG, Nicoletti F, Buttari F, Mercuri NB, Martino G, Furlan R, Centonze D. Interleukin-1 promotes long-term potentiation in patients with multiple sclerosis. Neuromolecular Med 2014; 16: 38-51.

22. MunkAS, Wang W, Bèchet NB, Eltanahy AM, ChengAX, Sigurdsson B, Benraiss A, Mäe MA, Kress BT, Kelley DH, Betsholtz C, Møllgård K, Meissner A, Nedergaard M, Lundgaard I. PDGF-B is required for development of the glymphatic system. Cell Rep 2019; 26: 2955 2969.

23. Musella A, Mandolesi G, Mori F, Gentile A, Centonze D. Linking synaptopathy and gray matter damage in multiple sclerosis. Mult Scler 2016; 22: 146-149.

24. Nelissen I, Gveric D, van Noort JM, Cuzner ML, Opdenakker G. PECAM-1 and gelatinase B coexist in vascular cuffs of multiple sclerosis lesions. Neuropathol Appl Neurobiol 2006; 32: 15-22.

25. Park E, Gallezot JD, Delgadillo A, Liu S, Planeta B, Lin SF, O'Connor KC, Lim K, Lee JY, Chastre A, Chen MK, Seneca N, Leppert D, Huang Y, Carson RE, Pelletier D. (11)C-PBR28 imaging in multiple sclerosis patients and healthy controls: test-retest reproducibility and focal visualization of active white matter areas. Eur J Nucl Med Mol Imaging 2015; 42: 1081-1092.

26. Paty DW, Moore GRW. Magnetic resonance imaging changes as living pathology in multiple sclerosis. In: Paty DW, Ebers GC (eds.). Multiple sclerosis. FA Davis, Philadelphia 1998; pp. 328-369.

27. Plant SR, Arnett HA, Ting JP. Astroglial-derived lymphotoxin-alpha exacerbates inflammation and demyelination, but not remyelination. Glia 2005; 49: 1-14.

28. Plog BA, Nedergaard M. The glymphatic system in central nervous system of health and disease: past, present, and future. Ann Rev Pathol 2018; 13: 379-394.

29. Rooney WD, Coyle PK. Recent advances in the neuroimaging of multiple sclerosis. Curr Neurol Neurosci Rep 2005; 5: 217-224.
30. Rosenberg GA. Matrix metalloproteinases and neuroinflammation in multiple sclerosis. Neuroscientist 2002; 8: 586-595.

31. Saha RN. Regulation of inducible nitric oxide synthase gene in glial cells. Antioxid Redox Signal 2006; 8: 929-947.

32. Sinclair C, Mirakhur M, Kirk J, Farrell M, McQuaid S. Up-regulation of osteopontin and alphaBeta-crystallin in the normal-appearing white matter of multiple sclerosis: an immunohistochemical study utilizing tissue microarrays. Neuropathol Appl Neurobiol 2005; 31: 292-303.

33. Sunnemark D, Eltayeb S, Nilsson M, Wallström E, Lassmann $\mathrm{H}$, Olsson T, Berg AL, Ericsson-Dahlstrand A. CX3CL1 (fractalkine) and CX3CR1 expression in myelin oligodendrocyte glycoproteininduced experimental autoimmune encephalomyelitis: kinetics and cellular origin. J Neuroinflamm 2005; 2: 17.

34. Thomas JL, Jacob L, Boisserand L Lymphatic system in central nervous system. Med Sci (Paris) 2019; 35: 55-61.

35. Traboulsee A, Dehmeshki J, Peters KR, Griffin CM, Brex PA, Silver N, Ciccarrelli O, Chard DT, Barker GJ, Thompson AJ, Miller DH. Disability in multiple sclerosis is related to normal appearing brain tissue MTR histogram abnormalities. Mult Scler 2003; 9: 566-573.

36. Traboulsee A, Zhao G, Li DK. Neuroimaging in multiple sclerosis. Neurol Clin 2005; 23: 131-148.

37. van Nierop GP, van Luijn MM, Michels SS, Melief MJ, Janssen M, Langerak AW, Ouwendijk WJ, Hintzen RQ, Verjans GM. Phenotypic and functional characterization of $\mathrm{T}$ cells in white matter lesions of multiple sclerosis patients. Acta Neuropathol 2017; 134: 383-401.

38. Villa A, Vegeto E, Poletti A, Maggi A. Estrogens, neuroinflammation and neurodegeneration. Endocr Rev 2016; 37: 372-402.

39. Wierzba-Bobrowicz T, Lewandowska E, Lechowicz W, Stępień T, Pasennik E. Quantitative analysis of activated microglia, ramified and damage of processes in the frontal and temporal lobes of chronic schizophrenics. Folia Neuropathol 2005; 43: 81-89.

40. Wojtera M, Sobów T, Kłoszewska I, Liberski PP, Brown DR, Sikorska B. Expression of immunohistochemical markers on microglia in Creutzfeldt-Jakob disease and Alzheimer's disease: morphometric study and review of the literature. Folia Neuropathol 2012; 50: 74-84. 